\title{
Dialogues: The Science and Power of Storytelling
}

\author{
๑Dendy A. Suzuki, ${ }^{1}$ @Mónica I. Feliú-Mójer, ${ }^{2,3}$ Uri Hasson, ${ }^{4}$ Rachel Yehuda, ${ }^{5}$ and $\odot$ Jean Mary Zarate ${ }^{6}$ \\ ${ }^{1}$ Center for Neural Science, New York University, New York, New York 10003, ${ }^{2}$ Ciencia Puerto Rico, San Juan, Puerto Rico 00919, ${ }^{3}$ iBiology, San Francisco, \\ California 94158, ${ }^{4}$ Department of Psychology and the Neuroscience Institute, Princeton University, Princeton, New Jersey 08540, ${ }^{5}$ Department of Psychiatry \\ and Neuroscience, Icahn School of Medicine at Mount Sinai, New York, New York 10029, and 'Nature Neuroscience, New York, New York $10004-1562$
}

Skillful storytelling helps listeners understand the essence of complex concepts and ideas in meaningful and often personal ways. For this reason, storytelling is being embraced by scientists who not only want to connect more authentically with their audiences, but also want to understand how the brain processes this powerful form of communication. Here we present part of a conversation between a group of scientists actively engaged with the practice and/or the science of storytelling. We highlight the brain networks involved in the telling and hearing of stories and show how storytelling is being used well beyond the realm of public communication to add a deeper dimension to communication with our students and colleagues, as well as helping to make our profession more inclusive.

Key words: outreach; inclusion; communication; science education

\section{Introduction}

It is now more urgent than ever that scientists take an active role in engaging with and educating the public about what they do as scientists, why they do it, and why it matters. It is in this context that many scientists hear about the craft of storytelling. The personal narrative detail that is often at the heart of a good story is one of the most powerful forms of communication that exists. Indeed, the craft of storytelling has enjoyed a renaissance of sorts with storytelling-based approaches being used in everything from promoting new startups, to inspiring creativity in the workplace, to business leadership strategy. This is because stories, and in particular personal stories, have the ability to illuminate fault lines, highlight oddities, and paint a picture of the past, present, and future that is both compelling and easily understandable.

The science community has also started to embrace the power of storytelling, as illustrated by the popularity of science storytelling organizations, such as The Story Collider, science storytelling socials at the Annual Society for Neuroscience (SFN) meeting, and SFN minisymposia devoted to the topic of storytelling. What follows here is part of a larger conversation among some of the members of the 2018 SFN minisymposium entitled "Telling stories of science." In anticipation of that minisymposium event, we present part of an interview-style conversation led by the chair of that session, Wendy Suzuki, and several of the symposium participants. The theme of the conversation is the science and power of storytelling. We start with what we know about the pattern of brain activation seen when we hear, view, or tell stories, based on the work of Prof. Uri Hasson. We then address the variety of ways that bench scientists (Prof. Rachel Yehuda) and science professionals (Dr. Mónica I. Feliú-Mójer and Dr. Jean Mary Zarate)

\footnotetext{
Received Aug. 31, 2018; revised Sept. 24, 2018; accepted Sept. 26, 2018.

The authors declare no competing financial interests.

Correspondence should be addressed to Dr. Wendy A. Suzuki, Department of Neural Science and Psychology, New York University, 4 Washington Place, Room 809, New York, NY 10003. E-mail: ws21@nyu.edu.

DOI:10.1523/JNEUROSCI.1942-18.2018

Copyright $\odot 2018$ the authors $\quad 0270-6474 / 18 / 389468-03 \$ 15.00 / 0$
}

have used storytelling in their professional lives. It is clear that storytelling is not only the focus of a growing body of cognitive neuroscientific exploration, but is already being used in a myriad of ways not only to educate the general public about the value of science, but to effectively convey essential information to our colleagues and students and, more broadly, to help improve the way science is done.

\section{The science of storytelling}

WS: Prof. Uri Hasson, why did you start looking at the brain activation associated with stories?

UH: I am interested in the neural mechanism underlying human communication. On a daily basis, I am able to communicate my thoughts, feeling, and intentions, using words, to other brains while at the same time being able to comprehend other brains' own spoken words. It occurred to us that stories are one of the most effective ways to communicate, so we started to look at what is going on in the listeners' brains as they listen to real-life spoken stories, and how their brains' responses relate to the responses observed in the storyteller's brain as she tells the story.

WS: What did you find?

UH: To my great surprise, we found that the neural activity in many cortical and subcortical areas was similar across all listeners, ranging from early auditory areas, to linguistic areas, to highorder areas in the parietal and frontal cortices. As expected, the intersubject neural similarity in early auditory areas was coupled to the acoustic properties of the spoken words. In contrast, the intersubject neural similarity in high-order areas, which mainly overlap with the default mode network (DMN), was decoupled from the acoustic features and was coupled to the story's narrative.

Further studies showed, for example, that the neural responses in the DMN were similar across Russian listeners who listened to a Russian story and English listeners who listened to a translated version of the same story, demonstrating that the same narrative can evoke similar responses, regardless of the linguistic system used to convey it (Honey et al., 2012). 
WS: Given that the same story can be understood in many different ways, how can all listeners exhibit similar brain responses when listening to a story?

UH: Excellent point! We hypothesized that the level of similarity in high-order areas across subjects is mediated by the level of shared understanding. To test this idea, we manipulated the level of shared understanding to the exact same narrative. Here we presented an ambiguous story to subjects. Then we ruined the ambiguity of the story, by telling half of the subjects one version of the story and the other half a different version (Yeshurun et al., 2017). We then measured the similarity in brain responses in the same high-order brain areas across subjects and found that the responses were more similar between subjects who had similar interpretation of the narrative relative to subjects with the opposing interpretation. Indeed, we could classify, with about $85 \%$ confidence, the subjects' interpretation based on the similarity of their brain responses to other subjects who were given the same version.

WS: So far, you've only told us about the listeners' brains. What is happening with the speaker's brain?

UH: When we compared the responses in the speaker's brain as she told a story to the responses in the listeners' brain as they listened to it, we found that the responses in the listeners' brain are coupled (correlated with a lag) to the responses in the speaker's brain (Stephens et al., 2010). Furthermore, the stronger the correlation (neural alignment) between the speaker's brain and listener's brain, the better the communication, as assessed with postscan comprehension tests.

WS: Fascinating! What do you think are the implications of these findings?

UH: We gather our ideas and belief from other brains we are connected to-show me your friends and I will tell you who you are-and from the social media we consume. Sadly, in this current time, there are forces that try to set us apart. We are living in a polarized world, in which people in our society are losing its common ground and drifting apart. Our task, as scientists and as storytellers, is to see how we can re-create and rebuild our common uniting story, while at the same time, preserving our personal stories and allowing each and every one of our unique voices to be heard.

\section{The power of storytelling}

We now turn to insights from some of our seasoned science storytellers. It seems that learning and sharing your science stories have affected and inspired all of them in both similar and unique ways. We often think about storytelling in the context of public speaking, but Prof. Rachel Yehuda explicitly uses it when giving regular science talks.

WS: How has storytelling helped you communicate with fellow scientists?

RY: I have always used storytelling to communicate science to my science colleagues because the backstory behind how a scientific question gets asked and answered is as interesting as the data generated. Engaging listeners in the scientific journey creates a stronger, more meaningful transfer of knowledge because it elicits participation and creates an intellectual investment and emotional bond between the speaker and the audience.

Details about the scientists' personal life can also be relevant to the scientific journey and questions being asked. Autobiographical and personal reflections may seem like the opposite of objective scientific data, but since I have started including more of these in presentations, I have been surprised by the synergy between anecdote and fact. Their combination appears to increase the salience of information to the public who are the ultimate consumers, and also funders through their tax dollars, of scientific information.

WS: Dr. Jean Zarate, how has science storytelling helped in your role as an editor?

JZ: I found that science storytelling changed the way I interact with my science colleagues in my role as editor at Nature Neuroscience. For my editorial duties, I tend to focus only on the science and determine whether the overarching messages in the paper are impactful and properly supported by the data and the interpretations. But by actually preparing and presenting at a science storytelling event myself, I was reminded about the human side to the science I read every day: that there are at least one or two personal stories motivating scientists' research interests and their career trajectories. And in sharing a story about my own scientific path, I slowly realized that revealing some details about my personal science trajectory, including sharing with the scientists my science passions, allowed the investigators and trainees to see a more human side to someone who was often regarded simply as "the editor." I think remembering that these more personal stories exist, on both sides, has influenced the way I interact with researchers when discussing their work and when helping them to communicate their findings and their implications more clearly to our readers.

WS: Dr. Zarate, has storytelling also affected the way you help your authors shape their introductions? If so, how?

JZ: Absolutely. As I prepared my story, I learned how to engage the audience quickly and tweak the text to avoid alienating people with statements that I thought were harmless. For scientific papers, the introduction is a perfect opener to engage the audience and get them excited to read your research or your review paper. If it takes nearly two pages to reach the research question or thesis statement or if the introduction is filled with technical jargon or casts certain work in an unfavorable light, then you will lose your audience quickly. So, I work with authors to present their ideas in a clear, succinct manner that communicates their ideas in a balanced and appropriate tone, so that the readers will keep reading.

WS: Dr. Mónica Feliú-Mójer, can you share your unique perspective on the power of storytelling and how you have used it in your career?

MF-M: As a kid, I devoured storybooks. When I wasn't reading, or making up my own stories, I was out and about in my rural home, catching lizards and finding rocks. Although I have always loved science and storytelling, I didn't connect the two until I was about to start my PhD.

After completing my undergraduate degree, I moved from Puerto Rico to Boston. Far from home, I longed for an opportunity to stay connected and give back to my community. I stumbled upon that opportunity in science storytelling, when I volunteered to write and edit popular science stories for Puerto Rico's main newspaper. During graduate school, telling the stories of Puerto Rican science and scientists empowered me to bring my whole self into research, and unapologetically connect my culture and science. Now, as a professional science communicator, I use storytelling as a tool for inclusion and social justice.

Originally, I thought that by leaving lab research to be a communicator, I would miss out on one of my favorite things about science: using my creativity to help me solve problems. But surprisingly, science storytelling has unleashed my creativity in unexpected ways. As a storyteller, I get to apply the process of discovery to find ways to make science compelling, or work with an animator to translate a concept into visuals. 
Today, I combine storytelling with my scientific and cultural backgrounds to make science more accessible and inclusive to communities that are underrepresented in, and excluded from, science. For example, I coproduce a series called "Background to Breakthrough" that is flipping the narrative about the value of diversity in science. Instead of telling the typical underdog story, the series looks at how the identities, culture, and background of underrepresented scientists fuel their ingenuity and approaches to problem solving. I also get to share my expertise by training young underrepresented scientists in science communication. Science storytelling has allowed me to meaningfully connect with myself and my communities. Telling science stories changed the course of my career, and I am a better scientist for it.

WS: Why do you think that personal element is so powerful especially when we as scientists speak to the public?

RY: Personal stories are not only humanizing, but if the scientist takes the time to convey potentially technical information in a more accessible manner, it is easier for the public to understand how the information is directly relevant to them. For example, my work is on the long-lasting impact of trauma exposure on oneself and possibly even one's descendants and involves neurobiological and molecular mechanisms. I have found that telling the story of how this work developed and presenting the work simply provides a greater reach and resonance than if the observations were presented as they are in scientific journals.

\section{Conclusion}

Storytelling is both a useful and fascinating topic to scientists from a multitude of perspectives. From a cognitive neuroscience perspective, it is of great interest to understand how this ancient form of communication engages and even entrains our brains in reproducible ways. From a practical perspective, it is of great interest to understand how storytelling can be used to help us improve both how we engage in science and expand who engages in science. There is much more we have to learn about how we as scientists can incorporate storytelling into our professional lives as we strive to make science more understandable, more inclusive, and ultimately, more beneficial to the world.

\section{References}

Honey CJ, Thompson CR, Lerner Y, Hasson U (2012) Not lost in translation: neural responses shared across languages. J Neurosci 32:1527715283. CrossRef Medline

Stephens GJ, Silbert LJ, Hasson U (2010) Speaker-listener neural coupling underlies successful communication. Proc Natl Acad Sci U S A 107: 14425-14430. CrossRef Medline

Yeshurun Y, Swanson S, Simony E, Chen J, Lazaridi C, Honey CJ, Hasson U (2017) Same story, different story. Psychol Sci 28:307-319. CrossRef Medline 